\title{
Facteurs d'Évolution de la Contribution Économique des Femmes au Niger
}

\author{
Par \\ Mahamane Ibrahima \\ Département de Démographie, Université de Montréal, Canada
}

\begin{abstract}
Résumé
Les discours politiques au Niger suggèrent une participation accrue des femmes dans le marché de l'emploi, mais les raisons de cette évolution (politique de la promotion de la femme, ou crise économique) restent mal connues. Cet article s'appuie sur des données des recensements de 1988 et 2001 pour examiner les changements intervenus dans les niveaux et déterminants de l'emploi des femmes au Niger. Les résultats montrent que le niveau d'activité de la femme nigérienne n'a que légèrement augmenté, mais varie selon le statut et la résidence. La crise économique n'aurait affecté l'activité féminine qu'en milieu urbain, alors que le milieu rural est influencé par les variations climatiques. Considéré comme un obstacle au travail féminin en Afrique Subsaharienne, le mariage favorise l'exercice d'une activité économique pour la femme en milieu rural mais n'a aucun effet en milieu urbain.
\end{abstract}

Mots clés : Déterminants de l'activité économique, Activité de la femme, promotion de la femme, Afrique, Niger.

\section{Abstract}

Public debates in Niger suggest a growing participation of women in the labor force, but the reasons underlying this trend (whether policy or economic conditions) remain poorly understood. This study uses census data from 1988 and 2001 to investigate the changes in the levels of determinants of women's employment in this country. The results show modest improvements in women's labor force participation, but participation is highly variable depending upon women's household headship or residence. Only in urban settings did the country's economic crisis impact women's labor force participation, while climatic problems matter most for rural residents. While it is often seen as limiting women's labor force involvement, marriage does in fact enhance labor force participation in rural but not in urban areas. 


\section{Introduction}

L'image de la femme africaine évolue sous l'influence des changements socioéconomiques et de la lutte pour la promotion de la femme. Au Niger, le début d'une réelle prise de conscience de ses droits par la femme a coïncidé avec la Conférence Nationale Souveraine (CNS) de 1991. A l'issue de celle-ci, la politique de promotion de la femme devrait atteindre les objectifs suivants : améliorer les conditions socio-économiques et juridiques, promouvoir l'éducation et l'alphabétisation, valoriser la productivité économique de la femme (Dunbar et Djibo, 1992). Plus de dix (10) ans après, les discours notent des progrès sensibles dans l'alphabétisation, l'élimination de plusieurs formes de discriminations à l'égard de la femme, et une présence accrue des femmes dans les sphères de décision politique et d'activité économique (Clignet, 1972; World Bank, 1998; Sow, 2000; United Nations, 2001; Adjamagbo et al., 2004; FeDDAF, 2004). Toutefois, l'évaluation des ces progrès et de leurs déterminants reste incomplète (Nunez, 1989; Anker, 1994; Chari, 2000; Sow, 2000; Dial, 2001; Adjamagbo et al., 2004; Unifem, 2005). Bien que les études aient examiné cette réduction des écarts entre hommes et femmes, peu de travaux ont analysé les différences entre les femmes elles-mêmes, et encore moins les déterminants de ces réductions. Pour combler cette lacune, le présent article examiner les changements intervenus dans les niveaux et déterminants de l'activité féminine, en s'appuyant sur des données de recensement, notamment de 1988 et 2001.
L'étude porte sur le Niger, l'un des pays les plus pauvres du monde, dans lequel le contrôle masculin de la plupart des activités féminines (Bisilliat, 1983; Schildkrout, 1983; Oppong, 1983) serait en train de se réduire. Le contexte nigérien est d'autant plus intéressant que sa population est en large majorité rurale $(85 \%)$ et musulmane (95\%).

\section{Revue de Littérature}

Les études antérieures sur l'activité des femmes suggèrent une implication croissante dans le marché de l'emploi, mais les déterminants de ces changements restent à élucider (Fapohunda, 1983; Bagchi, 1998; Sow, 2000; Dial, 2001; Adjamagbo et al., 2004; Unifem, 2005). Avec l'urbanisation, la modernisation, et la scolarisation des filles, l'activité économique féminine se différencie de plus spatialement. En milieu rural, l'activité de la femme se réduit souvent à l'agriculture et au travail domestique (Bisilliat, 1983; Fapohunda, 1983; Anker, 1994; World Bank, 1998; Unifem, 2005), sous un contrôle étroit du chef de famille (Dupire, 1960; Fapohunda, 1983; Schildkrout, 1983; Di Domenico, 1987; World Bank, 1998). Elle serait par ailleurs hiérarchiquement répartie entre les jeunes filles qui aident leurs mères, les femmes adultes s'occupant du bien-être de la famille et les aînées qui effectuent des activités d'ordre social (Bisilliat, 1983; Schildkrout, 1983).

En milieu urbain par contre, l'activité féminine est caractérisée par le système moderne basé sur le salariat et la 
qualification (Bisilliat, 1983; Fapohunda, 1983; Chari, 2000; Sow, 2000). Contrairement au milieu rural, ici, la femme dispose personnellement $\mathrm{du}$ revenu de son travail (Dupire, 1960; Fapohunda, 1983; Schildkrout, 1983; Di Domenico, 1987; World Bank, 1998). Ce serait donc surtout dans le contexte urbain que la promotion de la femme a gagné du terrain avec l'augmentation du nombre de femmes chefs de ménages. Par contre, certains auteurs estiment que la femme perdrait plus qu'elle ne gagne dans une économie moderne implantée dans un contexte de faible instruction. Dans la société moderne Songhay-Zarma du Niger par exemple, «les femmes perdraient leurs propres chaines d'appropriation qui leur permettraient de jouer leurs rôles reproducteurs avec la dignité requise dans leurs sociétés parce qu'elles sont effectivement rejetées hors du système économique dominant et de la sphère de décision, et négligées dans l'éducation et la formation» (Bisilliat 1983:106).

Les différences socioculturelles -l'ethnie et la religion notamment peuvent aussi influencer l'autonomie économique de la femme (Bisilliat, 1983; Schildkrout, 1983; Nunez, 1989; Sow, 2000; Dial, 2001; Adjamagbo et al., 2004; Adjamagbo et Antoine, 2004; Unifem, 2005). Au sein de certains groupes en effet, le mariage

\footnotetext{
$1 \overline{\text { Citation originale : «will lose their own channels }}$ of appropriation which, albeit ritual and symbolic and unacknowledged in the male culture, permitted them to carry out their reproductive roles with dignity required for their roles as socialisers» parce qu' elles sont effectivement «rejected in the dominant economic mode and in decision-making, neglected in the sphere of educati on and training».
}

limiterait l'autonomie économique de la femme en réduisant sa liberté de mouvement. Néanmoins Schwarz (1972) suggère que ces restrictions ne sont réelles que dans les sociétés où le mariage donne à la femme le droit d'être prise en charge totalement par le mari. Prenant l'exemple de certaines sociétés urbaines de la République Démocratique du Congo (Ex Zaïre), il conclut que le travail de la femme sert surtout à renflouer le budget du ménage en cas de défaillance du mari. Ceci corrobore les résultats de Di Domenico et al. (1987) qui parlent de pression sur la femme urbaine Yoruba (Nigeria) pour prendre en charge ses enfants et accumuler la richesse dans le but de prévenir un éventuel second mariage de son conjoint. Les effets du statut matrimonial sur le travail féminin dépendraient donc de la situation économique du conjoint de la femme ou de son ménage. Ainsi, les femmes vivant dans les ménages aisés auraient moins de pression de travailler que celles vivant dans les ménages pauvres. La réalité est toutefois plus complexe, dans la mesure où la pauvreté pourrait aussi limiter le travail des femmes en réduisant leurs capacités physiques et leur disponibilité (World Bank, 1999; Unifem, 2005).

D'autres facteurs à prendre en compte incluent l'alphabétisation (Rexroat, 1990) et le nombre d'enfants (World Bank, 1998). Schildkrout (1983) estime que l'implication des enfants dans les tâches domestiques permettrait à une femme d'exercer des activités en dehors du foyer, même si les résultats de Béguy (2004) à Lomé ne confirment pas cette thèse. Même 
en Occident où cela semblait être une évidence, Véron (1988) et Blanchet (1992) ont montré qu'en France, l'activité et la fécondité des femmes sont interdépendantes.

\section{Hypotheses}

Les évolutions économiques et sociales décrites plus haut suggèrent deux hypothèses. La première hypothèse suppose une augmentation de niveau d'activité des femmes au Niger entre 1988 et 2001, sous l'influence de la crise économique nigérienne et de la politique de la promotion de la femme. La seconde soutient que ces évolutions dépendent des plusieurs caractéristiques, notamment du niveau de revenus des ménages, de son statut (chef de ménage ou non) de la femme, et de son ethnie.

\section{Données et Méthodes}

En l'absence de données longitudinales appropriées (Kempeneers, 1992; Dial, 2001), nous utiliserons les données des échantillons de 10\% des recensements de 1988 et 2001 du Niger. Ceux-ci représentent la seule source de données d'envergure nationale qui contienne des informations sur l'activité féminine au Niger (Anker, 1994; Kempeneers, 1992). Même si les données sont disponibles pour toutes les femmes indépendamment de leur nationalité, notre analyse exclut les femmes non-nigériennes. Les dates des recensements retenus (1988 et 2001) sont chronologiquement pertinentes car le premier a eu lieu trois ans avant la Conférence Nationale Souveraine de 1991 au Niger (début de la phase active du mouvement féministe nigérien), tandis que le second a lieu dix ans après cet événement.

La variable dépendante est le statut d'emploi, qui prend les valeurs 1 si la femme travaille et 0 si non. Selon les deux derniers recensements du Niger, une femme est considérée comme travailleuse (ou occupée) si elle est âgée d'au moins 10 ans et déclare avoir exercé une activité économique au cours des six derniers mois précédant le recensement. En cas d'exercice de pluri-activité, l'activité la plus fréquente est retenue.

Les variables indépendantes d'intérêt incluent le statut familial, le niveau de vie du ménage, le statut matrimonial et l'appartenance ethnique. Les variables indépendantes de contrôle sont: l'âge, la taille du ménage, la région de résidence, la structure du ménage, le sexe du chef de ménage et l'alphabétisation de la femme. Les trois dernières variables de contrôle seront retenues uniquement pour le milieu urbain à cause d'un problème de faible effectif en milieu rural (moins de 5\% du total).

Le statut familial est mesuré par le lien de parenté avec le chef de ménage. Il comprend 5 modalités: chef de ménage, épouse, fille, autre parent et sans lien avec le chef de ménage. Le niveau de vie du ménage est un indicateur construit à partir des caractéristiques du ménage selon le milieu de résidence. Il a 3 modalités : aisé, moyen et pauvre. Nous regroupons les huit principales ethnies en 4 grands groupes sur 
une base régionale : Haoussa (Haoussa, Kanuri et Toubou), Djerma (Djerma, Gourmantché et Mossi), Peulh, Touareg (Touareg et Arabes). Le statut matrimonial comprend 4 modalités: monogame, polygame, veuve/divorcée et jamais mariée. La structure du ménage sera déterminée par la présence d'un enfant de moins de 6 ans, d'un jeune de 6-17 ans ou d'un adulte de 18-54 ans dans le ménage. La variable région comprend 5 modalités : le Centre, le Nord, l'Est, l'Ouest et la Capitale.

Nos analyses commencent par une description de l'évolution du statut et niveau d'activité de la femme entre 1988 et 2001, avant d'examiner les facteurs qui influencent la probabilité de travailler de la femme nigérienne pour chacune de ces deux années. Les analyses se feront séparément pour les milieux urbain et rural. L'analyse explicative se fera à l'aide de trois modèles logistiques pour chaque année et pour chaque milieu de résidence, dans le Tableau 2 (la partie A concerne le milieu rural et $B$, le milieu urbain).

\section{Résultats}

\section{Évolution du niveau d'activité des femmes}

Le niveau d'activité des femmes au Niger est faible, mais il a presque doublé en milieu rural (passant de 20,9\% en 1988 à $40,4 \%$ en 2001), alors qu'il n'a augmenté que de deux points en milieu urbain (passant de 21,5 à 23,7\% au cours de la période). Ces niveaux varient substantiellement en fonction des diverses caractéristiques de la femme. Le Tableau 1 présente ces évolutions selon le statut familial, le niveau de vie du ménage, la région de résidence, le statut matrimonial, le groupe ethnique et le groupes d'âge. Comme il fallait s'y attendre, les femmes chefs de ménage ont un taux d'activité plus élevé que les autres. Ce taux a légèrement augmenté entre 1988 et 2001 en milieu rural. Le niveau d'activité des femmes en milieu rural est relativement le même pour tous les niveaux de vie, mais a connu une forte augmentation entre 1988 et 2001. En milieu urbain, l'activité des femmes est plus courante dans les ménages aisés, mais elle a fortement chuté dans ce groupe entre 1988 et 2001; par contre, elle a légèrement augmenté dans des ménages moyens, et stagné dans les ménages pauvres.

D'importantes disparités régionales sont observées. La région Centre, capitale économique du pays, se démarque des autres régions par un niveau d'activité plus élevé, indépendamment du milieu de résidence. L'activité varie aussi selon le statut matrimonial dans les deux milieux de résidence. En milieu rural, l'activité a augmenté pour toutes les catégories, mais plus fortement chez les femmes mariées où on assiste à un doublement. En milieu urbain, le taux d'activité des célibataires a baissé celui des veuves/divorcées a légèrement augmenté.

Au plan socioculturel, les femmes Haoussa (ethnie majoritaire connue pour leur sens du commerce et de l'agriculture) ont les taux d'activité les plus élevés, alors que les Djerma (en milieu rural) et les Peuhles (en milieu urbain) ont les taux les plus bas, quelle que soit l'année. Ces taux ont augmenté dans chaque ethnie entre 
1988 et 2001 en milieu rural, surtout chez les Djerma où le niveau d'activité a plus que triplé. Les niveaux d'activité par ethnie sont restés presque stables entre 1988 et 2001 en milieu urbain. Enfin, les progrès dans l'activité féminine dépendent des groupes d'âge, ceux-ci étant le plus remarquables parmi les adultes (25-49 ans), avec un doublement entre 1988 et 2001. En milieu urbain, on note un léger progrès parmi les femmes de 25 ans et plus, mais une baisse chez les femmes âgées de moins de 25 ans.

Tableau 1 : Taux d'activité des femmes nigériennes selon certaines caractéristiques familiales et individuelles.

\begin{tabular}{|l|c|c|c|c|}
\hline \multicolumn{1}{|c|}{ Variables explicatives } & \multicolumn{2}{c|}{ Rural } & \multicolumn{2}{c|}{ Urbain } \\
\hline & $\mathbf{1 9 8 8}$ & $\mathbf{2 0 0 1}$ & $\mathbf{1 9 8 8}$ & $\mathbf{2 0 0 1}$ \\
Age & & & & \\
Moins 25 ans & 19,5 & 33,3 & 11,8 & 9,6 \\
$25-49$ ans & 21,5 & 43,2 & 27,2 & 31,3 \\
50 ans et plus & 20,0 & 34,3 & 28,5 & 30,4 \\
Niveau de signification & $* * *$ & $* * *$ & $* * *$ & $* * *$ \\
Lien de parenté & & & & \\
Chef de ménage & 44,5 & 61,9 & 50,5 & 53,2 \\
Épouse du chef & 19,2 & 40,9 & 18,7 & 25,2 \\
Fille du chef & 21,6 & 29,1 & 10,8 & 9,9 \\
Autre parent & 18,1 & 31,3 & 17,3 & 16,0 \\
Sans lien avec le chef & 19,5 & 39,1 & 21,4 & 17,8 \\
Niveau de signification & $* * *$ & $* * *$ & $* * *$ & $* * *$ \\
\hline État matrimonial & & & & \\
Mariée monogame & 18,2 & 40,1 & 19,2 & 25,1 \\
Mariée polygame & 22,4 & 45,2 & 21,3 & 28,6 \\
Veuve/divorcée & 23,5 & 35,7 & 36,0 & 37,7 \\
Jamais mariée & 21,3 & 27,9 & 11,3 & 9,6 \\
Niveau de signification & $* * *$ & $* * *$ & $* * *$ & $* * *$ \\
\hline Région & & & & \\
Nord & 17,7 & 25,3 & 17,6 & 19,7 \\
Est & 11,7 & 38,9 & 13,5 & 24,7 \\
Ouest & 12,1 & 31,4 & 16,1 & 23,2 \\
Centre & 49,1 & 61,2 & 44,0 & 28,8 \\
Capitale & - & - & 15,0 & 15,1 \\
Niveau de signification & $* * *$ & $* * *$ & $* * *$ & $* * *$ \\
\hline
\end{tabular}




\begin{tabular}{|l|c|c|c|c|}
\hline Ethnie & & & & \\
Djerma & 8,5 & 27,7 & 22,9 & 22,8 \\
Haoussa & 24,9 & 42,8 & 13,4 & 16,0 \\
Kanuri & 19,3 & 32,2 & 17,2 & 19,4 \\
Peulh & 20,5 & 29,9 & 19,2 & 20,8 \\
Touareg & 20,9 & 40,4 & 21,5 & 20,4 \\
Niveau de signification & $* * *$ & $* * *$ & $* * *$ & $* * *$ \\
\hline Sexe du chef de ménage & & & & \\
Féminin & - & - & 38,2 & 37,0 \\
Masculin & - & - & 18,2 & 21,1 \\
Niveau de signification & - & - & $* * *$ & $* * *$ \\
\hline Alphabétisée & - & & & \\
Oui & - & - & 16,3 & 20,1 \\
Non & - & & 20,9 & 25,8 \\
Niveau de signification & & & $* * *$ & \\
\hline Ensemble femmes 10 ans et plus & 20,9 & 40,4 & 21,5 & 23,7 \\
\hline
\end{tabular}

Source : Traitement 10\% des recensements Niger, 1988 et 2001.

L'analyse logistique bivariée (modèle M0 du tableau 2) présente les effets bruts des variables d'intérêt. Elle souligne l'effet prédominant du statut familial : être chef de ménage augmente au moins de trois fois l'activité (sauf en milieu rural en 2001 où le rapport de côte est de 2,34). L'hypothèse relative au niveau de vie du ménage est en partie infirmée. En milieu rural, une femme non pauvre a entre 8 et $34 \%$ plus de chance de travailler qu'une femme pauvre en 1988 et $15-16 \%$ plus de chance en 2001. Cette hypothèse se vérifie néanmoins en milieu urbain en 1988 où une femme vivant dans un ménage moyen a $26 \%$ moins de chance de travailler que celle qui vit dans un ménage pauvre. Au niveau bivarié, l'effet du statut matrimonial dépend de la résidence et de l'année. En milieu rural, les monogames ont moins de chance de travailler que les autres en 1988; alors qu'en 2001, les mariées ont plus de chance de travailler que les non mariées, bien que les polygames aient plus de chance de travailler que les monogames. En milieu urbain, comme on s'y attendait, les mariées monogames ont des niveaux d'activités moindres que les autres femmes, à l'exception des célibataires. Les résultats montrent aussi que les femmes Haoussa ont plus de chance de travailler que celles des autres groupes ethniques. Mais en milieu urbain, les écarts interethniques se sont réduits entre 1988 et 2001. En milieu rural, par contre, il y a réduction de l'écart avec les Djerma mais une augmentation de celui avec les Peuhles et Touarègues.

L'analyse multivariée montre les effets nets des différentes variables (modèle M1) ainsi que certaines interactions (modèle M2). Il en ressort quelques constats intéressants qui 
soulignent la différence entre milieux rural et urbain. Comme dans l'analyse bivariée, le statut familial présente toujours les effets les plus élevés. Toutefois, l'introduction de contrôles statistiques augmente l'effet du statut de chef de ménage en milieu rural, mais le diminue en milieu urbain. Cette situation s'expliquerait par la variation dans l'autonomie des femmes chef de ménage selon le milieu de résidence. En milieu rural, où le phénomène est rare, une femme chef de ménage serait assez autonome avec ou sans conjoint. Par contre, en milieu urbain, les femmes chefs de ménage sont souvent des femmes mariées à des polygames et qui habitent des maisons séparées de celles de leurs coépouses.

Lee effets du niveau de vie varient aussi selon la résidence. Il n'y a pas de différence significative liée au niveau de vie parmi les femmes rurales : même les différences notées en 1988 ont disparu en 2001. En milieu urbain, par contre, la différence entre femmes aisées et pauvres s'est accrue entre 1988 et 2001. En 1988, les femmes vivant dans des ménages moyens ont moins de chance de travailler que celles des ménages pauvres. La situation reflète bien la réalité des années 1980, et son boom économique qui permettait à de nombreuses femmes de compter sur une prise en charge par le mari ou tout autre parent. En 2001, par contre, la situation a changé, et beaucoup de femmes, désireraient désormais travailler pour prendre en charge certaines de leurs dépenses non couvertes par les hommes. Quant aux femmes vivant dans les ménages pauvres, leurs chances de travailler s'amenuiseraient à cause de la montée du chômage, de l'absence de formation et du manque de temps matériel pour faire autre chose que les travaux domestiques. Ceci rejoint le constat de l'Unifem (2005) qui suppose que la pauvreté d'un ménage pourrait être un obstacle au travail de la femme. En milieu rural, où l'activité principale est l'agriculture, les chances de travailler des femmes ne varient pas selon le niveau de vie du ménage car il s'agit d'une activité saisonnière.

Les effets de l'ethnie varient très fortement en fonction du milieu de résidence. Les Djerma ont moins de chance de travailler que les autres, quels que soit l'année et le milieu de résidence. Paradoxalement, l'activité des Djerma ont augmenté dans le temps pendant que celle des autres groupes ethniques baissait en milieu rural. Une explication serait d'ordre climatique. Après la famine de 1984-85, la région du Zarmaganda (peuplée essentiellement de Djerma) serait devenue hostile à toute pratique agricole et l'exode concernait aussi bien les hommes que les femmes de cette région. Au milieu des années 1990, il y a eu un développement d'ONGs autour de la Capitale où sont développées des activités génératrices de revenu en faveur des femmes. La situation des femmes Peuhles et Touareg serait une suite logique de la perte de leur bétail suite aux sécheresses et famines et d'une sédentarisation non intégrée dans leurs mœurs. Le départ des hommes et la difficulté de reconversion à d'autres activités ont réduit leurs chances de travailler, comme on le voit en 2001. 
Contrairement au milieu rural, la situation en milieu urbain est caractérisée par une similarité des tendances entre 1988 et 2001. Les écarts entre les Haoussa et les autres ethnies restent les mêmes quel que soit le modèle. Ce qui montre que les différences interethniques n'ont pas changé entre 1988 et 2001. Ce résultat indique surtout que, pour le milieu urbain, l'ethnie ne permet pas d'apprécier les différences socioculturelles des femmes.

Compte tenu de l'importance du statut matrimonial dans la littérature, nous avons examiné son interaction avec d'autres variables. La même chose a été faite avec l'âge et la région de résidence (Modèles M2). En milieu urbain, les femmes alphabétisées du Nord et de la Capitale ont plus de chance de travailler que celles qui vivent au Centre du pays. Ce résultat est conforme à une certaine réalité nigérienne où le Nord et la Capitale sont restés longtemps les pourvoyeurs d'emplois pour les qualifiés pendant les périodes de croissance économique. En milieu rural, les effets d'interactions les plus importants sont ceux liant la région au statut matrimonial. Ils indiquent qu'en réalité, les femmes non mariées des régions Nord et Est, et les veuves chefs de familles ont plus de chance de travailler que leurs homologues du Centre et mariées. Les différences selon le statut matrimonial au sein des ethnies sont remarquables en milieu rural et non significatives en milieu urbain. Les interactions montrent que les Haoussa non mariées ont moins de chance de travailler que les mariées. En effet, au sein de cette ethnie, les jeunes filles ont le devoir d'aider leurs mères avant le mariage; les femmes âgées sont prises en charge par les adultes et la femme mariée a le devoir de prendre soin de la famille. Chez les Djerma, les femmes non mariées ont plus de chance de travailler que les mariées, mais avec une différence non significative entre les célibataires et les mariées en 2001. Ce résultat confirme celui trouvé par Bisilliat (1983), selon lequel, dans la société Djerma, seules les femmes veuves ou ayant plus de 50 ans ont la possibilité de travailler librement. Chez les Peuhles, ce sont seulement les célibataires qui ont plus de chance de travailler que les mariées en 1988, alors qu'il n'y a pas de différence significative entre mariées et non mariées en 2001. Dans la tradition peuhle, tout comme chez les haoussas, la jeune fille s'occupe de tous les travaux extérieurs à la maison en remplacement de sa mère à partir de 14 ans. C'est par là qu'elle fait son apprentissage pour sa fonction future d'épouse et de mère. C'est le contraire chez les Touarègues chez qui on n'observe pas de différence significative entre mariées et non mariées en 1988; alors qu'en 2001, les premières ont moins de chance de travailler que les secondes.

Notons enfin que la présence d'une personne de plus de 6 ans a un effet positif sur le travail de la femme (non significatif en 1988) en milieu urbain, quelle que soit l'année. Celle d'un enfant de moins de 6 ans a un effet négatif. Ce résultat confirme en partie l'hypothèse de la relation négative entre le travail d'une femme et la présence d'un enfant à charge généralement observée en Occident, malgré certaines infirmations (Véron, 1988). 
African Population Studies Vol. $22 n^{\circ}$ 2/Etude de la Population Africaine Vol. $23 N^{*} 1$

Tableau 2 : Rapports de côtes des variables explicatives sur la probabilité d'être active chez les femmes nigériennes

\begin{tabular}{|c|c|c|c|c|c|c|}
\hline \multicolumn{7}{|c|}{ A : Milieu rural } \\
\hline \multirow[t]{2}{*}{ Variables explicatives } & \multicolumn{3}{|c|}{1988} & \multicolumn{3}{|c|}{2001} \\
\hline & $\mathbf{M}_{0}$ & $\mathbf{M}_{1}$ & $\mathbf{M}_{2}$ & $\mathbf{M}_{0}$ & $\mathbf{M}_{1}$ & $\mathbf{M}_{2}$ \\
\hline $\begin{array}{l}\text { Lien de parenté (Épouse) } \\
\text { Chef de ménage } \\
\text { Fille } \\
\text { Autre parent } \\
\text { Sans lien avec le chef }\end{array}$ & $\begin{array}{c}3,35 * * * \\
1,16 * * * \\
0,93 * * * \\
1,02\end{array}$ & $\begin{array}{c}4,84 * * * \\
1,20 * * * \\
1,01 \\
1,10 * * *\end{array}$ & $\begin{array}{l}3,73 * * * \\
1,31 * * * \\
1,16 * * * \\
1,12 * * *\end{array}$ & $\begin{array}{l}2,34 * * * \\
0,59 * * * \\
0,66 * * * \\
0,93 * * *\end{array}$ & $\begin{array}{c}3,72 * * * \\
0,97 \\
0,92 * * * \\
0,96 *\end{array}$ & $\begin{array}{c}2,97 * * * \\
0,98 \\
0,95 * * * \\
0,97 * \\
\end{array}$ \\
\hline $\begin{array}{l}\text { Niveau de vie (pauvre) } \\
\text { Aisé } \\
\text { Moyen }\end{array}$ & $\begin{array}{l}1,08 * * * \\
1,34 * * *\end{array}$ & $\begin{array}{c}0,96 \\
0,94 * * *\end{array}$ & $\begin{array}{c}0,96 \\
0,94 * * *\end{array}$ & $\begin{array}{l}1,15^{* * * *} \\
1,16^{* * *}\end{array}$ & $\begin{array}{l}0,94 \\
1,01\end{array}$ & $\begin{array}{l}0,95 \\
1,01\end{array}$ \\
\hline $\begin{array}{l}\text { Statut matrimonial (Mono) } \\
\text { Polygame } \\
\text { Veuve/divorcée } \\
\text { Jamais mariée }\end{array}$ & $\begin{array}{l}1,29 * * * \\
1,37 * * * \\
1,21 * * *\end{array}$ & $\begin{array}{l}1,09 * * * \\
1,17 * * * \\
1,38 * * *\end{array}$ & $\begin{array}{c}1,07 * * * \\
0,61 * * \\
0,72 * * *\end{array}$ & $\begin{array}{l}1,23 * * * \\
0,83 * * * \\
0,58 * * *\end{array}$ & $\begin{array}{c}1,02 \\
0,80 * * * \\
0,75 * * *\end{array}$ & $\begin{array}{c}1,01 \\
0,52 * * * \\
0,50 * * *\end{array}$ \\
\hline $\begin{array}{l}\text { Ethnie (Haoussa) } \\
\text { Djerma } \\
\text { Peulh } \\
\text { Touareg } \\
\end{array}$ & $\begin{array}{l}0,28 * * * \\
0,72 * * * \\
0,78 * * *\end{array}$ & $\begin{array}{c}0,39 * * * \\
0,79 * * * \\
0,95 * * \\
\end{array}$ & $\begin{array}{l}0,35 * * * \\
0,79 * * * \\
0,92 * * *\end{array}$ & $\begin{array}{l}0,51 * * * \\
0,63 * * * \\
0,57 * * *\end{array}$ & $\begin{array}{l}0,54 * * * \\
0,60 * * * \\
0,80 * * *\end{array}$ & $\begin{array}{l}0,53 * * * \\
0,60 * * * \\
0,75 * * *\end{array}$ \\
\hline $\begin{array}{l}\text { Région (Centre) } \\
\text { Nord } \\
\text { Est } \\
\text { Ouest }\end{array}$ & $\begin{array}{l}0,22 * * * \\
0,14 * * * \\
0,14 * * *\end{array}$ & $\begin{array}{l}0,17 * * * \\
0,12 * * * \\
0,21 * * *\end{array}$ & $\begin{array}{l}0,14 * * * \\
0,10 * * * \\
0,20 * * *\end{array}$ & $\begin{array}{l}0,25 * * * \\
0,46 * * * \\
0,33 * * *\end{array}$ & $\begin{array}{l}0,23 * * * \\
0,45 * * * \\
0,49 * * *\end{array}$ & $\begin{array}{l}0,17 * * * \\
0,40 * * * \\
0,45 * * *\end{array}$ \\
\hline $\begin{array}{l}\text { Age (Moins de } 25 \text { ans) } \\
25-49 \text { ans } \\
50 \text { ans et plus }\end{array}$ & $\begin{array}{c}1,13 * * * \\
1,02 \\
\end{array}$ & $\begin{array}{l}1,18 * * * \\
0,89 * * *\end{array}$ & $\begin{array}{l}1,26 * * * \\
0,81 * * *\end{array}$ & $\begin{array}{l}1,52 * * * \\
1,05 * * *\end{array}$ & $\begin{array}{c}1,33 * * * \\
0,95\end{array}$ & $\begin{array}{l}1,32 * * * \\
0,84 * * *\end{array}$ \\
\hline Taille du ménage & $1,00 * * *$ & $0,99 * * *$ & $0,99 * * *$ & $1,00 * * *$ & 1,00 & 1,00 \\
\hline Taille au carré & - & $1,00 * * *$ & $1,00 * * *$ & - & 1,00 & $1,00 *$ \\
\hline Chef ménage*veuve & - & - & $1,84 * * *$ & - & - & $1,68 * * *$ \\
\hline Djerma*veuve & - & - & $1,65 * * *$ & - & - & $1,26 * * *$ \\
\hline Peulh*veuve & - & - & $0,76 * * *$ & - & - & NS \\
\hline Touareg*veuve & - & - & NS & - & - & $1,19 * * *$ \\
\hline Djerma*célibataire & - & - & $1,27 * * *$ & - & - & NS \\
\hline Peulh* célibataire & - & - & $1,12 *$ & - & - & NS \\
\hline Touareg* célibataire & - & - & NS & - & - & $1,15 * * *$ \\
\hline Nord*veuve & - & - & $2,38 * * *$ & - & - & $2,08 * * *$ \\
\hline Est*veuve & - & - & $2,18 * * *$ & - & - & $1,11 *$ \\
\hline Ouest*veuve & - & - & NS & - & - & $1,29 * * *$ \\
\hline Nord*célibataire & - & - & $3,51 * * *$ & - & - & $2,94 * * *$ \\
\hline Est*célibataire & - & - & $2,38 * * *$ & - & - & $1,16 * * *$ \\
\hline Ouest*célibataire & - & - & $1,27 * *$ & - & - & $1,46 * * *$ \\
\hline Nord*Femme2549 & - & - & $0,83 * * *$ & - & - & NS \\
\hline Est*Femme2549 & - & - & NS & - & - & $1,12 * * *$ \\
\hline Ouest*Femme2549 & - & - & NS & - & - & $0,95^{*}$ \\
\hline Nord*Femme50plus & & & $0,85^{*}$ & & & $1,15 * *$ \\
\hline Est*Femme50plus & & & NS & & & $1,20 * * *$ \\
\hline Ouest*Femme50plus & & & $1,53 * * *$ & & & $1,21 * * *$ \\
\hline Effectif correspondant & - & 186087 & 186087 & - & 271359 & 271359 \\
\hline
\end{tabular}

112 


\begin{tabular}{|c|c|c|c|c|c|c|}
\hline \multicolumn{7}{|c|}{ B : Milieu urbain } \\
\hline \multirow[t]{2}{*}{ Variables explicatives } & \multicolumn{3}{|c|}{1988} & \multicolumn{3}{|c|}{2001} \\
\hline & $\mathbf{M}_{0}$ & $\mathbf{M}_{1}$ & $\mathbf{M}_{2}$ & $\mathbf{M}_{0}$ & $\mathbf{M}_{1}$ & $\mathbf{M}_{2}$ \\
\hline $\begin{array}{l}\text { Lien de parenté (Épouse CM) } \\
\text { Chef de ménage } \\
\text { Fille } \\
\text { Autre parent } \\
\text { Sans lien avec le chef }\end{array}$ & $\begin{array}{c}4,43 * * * \\
0,53 * * * \\
0,91 * \\
1,19 * * *\end{array}$ & $\begin{array}{c}2,95 * * * \\
0,87 \\
1,07 \\
1,42 * * * \\
\end{array}$ & $\begin{array}{c}3,07 * * * \\
0,90 \\
1,09 \\
1,44 * * * \\
\end{array}$ & $\begin{array}{l}3,37 * * * \\
0,32 * * * \\
0,56 * * * \\
0,64 * * *\end{array}$ & $\begin{array}{c}2,08 * * * \\
0,71 * * * \\
0,81 * * * \\
0,95\end{array}$ & $\begin{array}{c}2,13 * * * \\
0,73 * * * \\
0,80 * * * \\
0,93\end{array}$ \\
\hline $\begin{array}{l}\text { Niveau de vie (pauvre) } \\
\text { Aisé } \\
\text { Moyen }\end{array}$ & $\begin{array}{l}1,95 * * * \\
0,74 * * *\end{array}$ & $\begin{array}{c}0,99 \\
0,83 * * *\end{array}$ & $\begin{array}{c}0,95 \\
0,84 * * *\end{array}$ & $\begin{array}{l}1,39 * * * \\
1,08 * * *\end{array}$ & $\begin{array}{l}1,30 * * * \\
1,10 * * *\end{array}$ & $\begin{array}{c}1,30 * * \\
1,09 * * *\end{array}$ \\
\hline $\begin{array}{l}\text { Statut matrimonial (Monog) } \\
\text { Polygame } \\
\text { Veuve/divorcée } \\
\text { Jamais mariée }\end{array}$ & $\begin{array}{l}1,14 * * * \\
2,37 * * * \\
0,54 * * *\end{array}$ & $\begin{array}{c}0,98 \\
1,27 * * * \\
0,92 \\
\end{array}$ & $\begin{array}{c}1,00 \\
0,89 \\
1,61 * * *\end{array}$ & $\begin{array}{l}1,19 * * * \\
1,80 * * * \\
0,31 * * *\end{array}$ & $\begin{array}{c}0,94 \\
1,11^{*} \\
0,66^{* * *} \\
\end{array}$ & $\begin{array}{l}0,97 \\
0,86 \\
0,93 \\
\end{array}$ \\
\hline $\begin{array}{l}\text { Ethnie (Haoussa) } \\
\text { Djerma } \\
\text { Peulh } \\
\text { Touareg } \\
\end{array}$ & $\begin{array}{l}0,52 * * * \\
0,70 * * * \\
0,83 * * *\end{array}$ & $\begin{array}{c}0,69 * * * \\
0,79 * * * \\
0,98 \\
\end{array}$ & $\begin{array}{c}0,66 * * * \\
0,75 * * * \\
0,98 \\
\end{array}$ & $\begin{array}{l}0,65 * * * \\
0,82 * * * \\
0,89 * * *\end{array}$ & $\begin{array}{c}0,81 * * * \\
0,86 * * \\
0,97 \\
\end{array}$ & $\begin{array}{c}0,71 * * * \\
0,78 * * * \\
0,97 \\
\end{array}$ \\
\hline $\begin{array}{l}\text { Région de résidence (Centre) } \\
\text { Nord } \\
\text { Est } \\
\text { Ouest } \\
\text { Capitale } \\
\end{array}$ & $\begin{array}{l}0,27 * * * \\
0,20 * * * \\
0,24 * * * \\
0,22 * * *\end{array}$ & $\begin{array}{l}0,20 * * * \\
0,16 * * * \\
0,26 * * * \\
0,23 * * *\end{array}$ & $\begin{array}{l}0,13 * * * \\
0,12 * * * \\
0,27 * * * \\
0,15 * * *\end{array}$ & $\begin{array}{l}0,61 * * * \\
0,81 * * * \\
0,74 * * * \\
0,44 * * *\end{array}$ & $\begin{array}{l}0,56 * * * \\
0,79 * * * \\
0,74 * * * \\
0,46 * * *\end{array}$ & $\begin{array}{l}0,51 * * * \\
0,74 * * * \\
0,71 * * * \\
0,33 * * *\end{array}$ \\
\hline $\begin{array}{l}\text { Age (Moins de } 25 \text { ans) } \\
25-49 \text { ans } \\
50 \text { ans et plus }\end{array}$ & $\begin{array}{l}2,91 * * * \\
3,11 * * *\end{array}$ & $\begin{array}{l}2,77 * * * \\
1,92 * * *\end{array}$ & $\begin{array}{l}2,90 * * * \\
2,13 * * *\end{array}$ & $\begin{array}{l}4,30 * * * \\
4,11 * * *\end{array}$ & $\begin{array}{l}2,96 * * * \\
2,38 * * *\end{array}$ & $\begin{array}{l}2,81 * * * \\
2,30 * * *\end{array}$ \\
\hline Alphabétisée & $0,74 * * *$ & $1,65 * * *$ & $1,51 * *$ & $0,69 * * *$ & $1,28 * * *$ & 1,00 \\
\hline $\begin{array}{l}\text { Sexe du chef de ménage (Mas) } \\
\text { Féminin }\end{array}$ & $2,86 * * *$ & $1,51 * * *$ & $1,50 * * *$ & $2,22 * * *$ & $1,61 * * *$ & $1,62 * * *$ \\
\hline Présence Enfant $<6$ ans & $0,77 * * *$ & $0,88 * * *$ & $0,86 * * *$ & $0,82 * * *$ & $0,88 * * *$ & $0,88 * * *$ \\
\hline Présence Jeune 6-18 ans & $0,75 * * *$ & 1,07 & 1,06 & $0,73 * * *$ & $1,12 *$ & $1,13 * *$ \\
\hline Présence Adulte 18-54 & 0,90 & $1,30 * * *$ & $1,30 * * *$ & $0,83 * * *$ & $1,30 * * *$ & $1,32 * * *$ \\
\hline Taille du ménage & $0,97 * * *$ & 1,00 & 1,00 & $0,96 * *$ & $1,01 * * *$ & $1,02 * * *$ \\
\hline Taille au carré & - & 1,00 & 1,00 & - & $1,00 * * *$ & $1,00 * * *$ \\
\hline Polygame*alphabétisée & - & - & 0,85 & - & - & NS \\
\hline Veuve*alphabétisée & & & $0,64 * *$ & & & NS \\
\hline Célibataire* alphabétisée & - & - & $0,13 * * *$ & - & - & $0,36^{* * *}$ \\
\hline Nord*veuve & - & - & $2,60 * * *$ & - & - & $1,42 * * *$ \\
\hline Est*veuve & - & - & $1,78 * * *$ & - & - & $1,43^{* *}$ \\
\hline Ouest*veuve & - & - & NS & - & - & NS \\
\hline Capitale*veuve & - & - & $1,79 * * *$ & - & - & $1,42 * * *$ \\
\hline Nord*célibataire & - & - & $1,98 * * *$ & - & - & NS \\
\hline Capitale*célibataire & - & - & NS & - & - & $1,20 *$ \\
\hline Nord* alphabétisée & - & - & $2,21 * * *$ & - & - & NS \\
\hline Est* alphabétisée & - & - & $1,87 * * *$ & - & - & $1,29 * *$ \\
\hline Ouest* alphabétisée & - & - & $1,65 * *$ & - & - & $1,27 *$ \\
\hline Capitale* alphabétisée & - & - & $3,26 * * *$ & - & - & $2,31 * * *$ \\
\hline Djerma*alphabétisée & & & NS & & & $1,35^{* * *}$ \\
\hline Peul*alphabétisée & & & NS & & & $1,42 * * *$ \\
\hline Effectif correspondant & - & 28441 & 28441 & - & 52766 & 52766 \\
\hline
\end{tabular}

Source : Traitement 10\% des recensements Niger, 1988 et 2001.

Signification : $:<5 \%$; ** : p<1\%; ***: $\mathrm{p}<0,1 \%$; MR : Modalité de référence 


\section{Résume et Discussion}

Nos analyses de l'activité des femmes au Niger montrent un doublement du niveau d'activité en milieu rural et une stagnation en milieu urbain. L'augmentation en milieu rural s'expliquerait par l'exode rural des hommes adultes causé non seulement par la rareté des terres cultivables mais aussi par les sécheresses cycliques. Ce changement pourrait aussi refléter la modification dans la définition de l'activité féminine lors du dernier recensement au Niger. La situation en milieu urbain pourrait s'expliquer par la crise économique qui réduit les opportunités d'emploi, celles-ci étant de plus en plus limitées au secteur informel jusque-là mal saisi lors des collectes de données.

Les résultats relatifs à notre seconde hypothèse sont mitigés. Ainsi, l'effet du niveau de vie du ménage n'est pas vérifié en milieu rural. En milieu urbain, la propension à travailler est positivement associée au niveau de vie du ménage, les ménages semblant ainsi être exclus de la réussite économique. C'est une des conséquences du programme de promotion de la femme qui a plus développé son côté visible en milieu urbain. A travers ce programme, de plus en plus de femmes ont eu accès au crédit pour réaliser des activités génératrices de revenu. En milieu urbain, la scolarisation et la formation a permis à certaines de trouver un emploi, mais ces opportunités n'apparaissent pas encore en milieu rural. Pendant ce temps, la crise a considérablement réduit le pouvoir d'achat des hommes qui perdent leurs activités principales et se retrouvent au chômage. La femme devient ainsi, et par la force des choses, la responsable économique du ménage en plus de son rôle d'éducatrice des enfants.

L'effet du statut familial est confirmé, sauf qu'il reflèterait plutôt une adaptation de crise plus qu'une évolution dans le statut de la femme. En effet, les différences entre chef de ménage et son épouse viennent du fait qu'au Niger, il revient en principe à l'homme de prendre en charge son épouse conformément à un engagement pris lors de la cérémonie du mariage religieux. La femme mariée nigérienne n'était pas obligée de travailler pour subvenir à ses besoins ou de contribuer aux dépenses du ménage. Aujourd'hui, le rôle économique de la femme nigérienne dans son ménage serait capital. L'absence de différence significative selon le niveau de vie de vie en 2001 en milieu rural pourrait suggérer l'importance des échanges de ressources entre familles nucléaires.

Le statut matrimonial affecte l'activité à travers d'autres variables telles que le statut familial, l'ethnie et la région de résidence. Contrairement à ce qui est attendu, l'effet du mariage est positif là où la femme est la moins alphabétisée, où le mariage est le plus précoce et où la religion pratiquée par la majorité (l'islam) est la moins tolérante, c'est-à-dire au Centre du pays. Par ailleurs, les femmes mariées ont moins de chance de travailler dans les régions du pays où la femme serait plus libre, son mariage moins précoce et où elle 
Mahamane Ibrahima: Facteurs d'Évolution de la Contribution Économique des Femmes au Niger

a plus de chance d'être alphabétisée.

Ce paradoxe indique que l'effet du mariage pourrait lui aussi résulter des effets d'autres variables. En effet, au Niger, le travail de la femme est plus apprécié s'il ne l'éloigne pas de sa famille. Au Centre du pays, les principales activités sont l'agriculture et le commerce. La femme pratique ces activités dans un cadre familial au sein duquel elle jouit d'une liberté relative. Dans les autres régions, l'agriculture et l'élevage dominent les activités, mais leur pratique y est difficile car les champs et les pâturages sont souvent à plusieurs kilomètres des villages ou des campements, ce qui réduirait la participation des femmes. La faible propension à travailler de la femme mariée montre que la politique de la promotion de la femme n'aurait donc pas eu les effets escomptés au Niger, ou du moins en partie. Les différences ethniques, marquées en milieu rural, s'estompent en milieu urbain, une homogénisation attribuable au processus de " modernisation 》 des populations urbaines.

$\mathrm{Au}$ total, la politique de la promotion de la femme au Niger, dix ans après la Conférence Nationale Souveraine qui a vu le départ du mouvement féminin, ne se traduit pas encore par une amélioration nette de l'activité économique de la femme. Son impact se limiterait encore peut être à d'autres sphères et ne toucherait que certaines catégories de femmes: les alphabétisées, les citadines et les non mariées chefs de ménages. La crise économique aurait pu donner un peu plus de pouvoir économique à la femme en réduisant le pouvoir d'achat des hommes. Mais cela n'apparait qu'en milieu rural pour des raisons autres que la crise économique qui sévit en milieu urbain. De manière générale, les caractéristiques socio-économiques (région de résidence, niveau de vie du ménage) et culturelles (statut matrimonial, ethnie) déterminent partiellement les chances de travailler d'une femme au Niger. Le statut matrimonial joue un rôle important mais complexe, et c'est surtout le statut familial de la femme qui détermine de manière la plus significative sa propension à travailler quel que soit le milieu de résidence. Les résultats trouvés reflèteraient la conception sociale du travail de la femme puisque l'épouse du chef de ménage a moins de chance de travailler que celle qui se déclare chef du ménage. Au vu de nos résultats, la politique de la promotion de la femme doit être repensée au Niger car, dans ce pays à $85 \%$ rural et $88 \%$ analphabète, la femme n'est valorisée, et ne se sent elle-même valorisée, que si elle est responsable de son foyer et de ses enfants dans les normes propres à son contexte. La crise économique a certes accru la visibilité du rôle économique des femmes urbaines, mais peut être augmenté sa dépendance en même temps. La promotion de l'activité féminine devrait donc inclure également une valorisation de son activité domestique dans les enquêtes et comptes nationaux. Nos résultats montrent enfin les insuffisances des données de recensements pour mesurer l'impact réel de la promotion de la femme au Niger et de la crise économique au Niger. Dest études plus affinées, basées sur des données longitudinales et plus riches en contenu 
sont nécessaires pour mieux comprendre les changements dans ce secteur fondamental.

\section{Bibliographie}

Adjamagbo, A. et Antoine, P., 2004, Etre femme «autonome» dans les capitales africaines : le cas de Dakar et Lomé, Dial, Document de travail DT/2004/03, 13 pages.

Adjamagbo, A., Antoine, P. et Dial, F.B., 2004, «Le dilemme des dakaroises : entre travailler et «bien travailler», pp. 247-272. In Gouverner le Sénégal : entre ajustement structurel et développement durable, Edited by Diop M-C., Paris, Karthala.

Anker R., 1994, «Measuring Women's Participation in the African Labour Force», pp. 64-75. In Gender, Work and Population in Sub-Saharan Africa, Edited by Adepoju et Oppong.

Antoine, P. et Nanitelanio, J., 1996, «Nouveaux statuts féminins et urbanisation en Afrique». GENUS XLVI, (3-4):17-30.

Bagchi A. D., 1998, Women at Work: A Study of the Factors Influencing Women's Employment, 1972-1985, CDE
Working Paper $\mathrm{N}^{\circ} .98-27,50 \mathrm{p}$.

Béguy, D., 2004, Emploi et fécondité à Lomé : Quelles interactions ? DIAL, Document de travail, DT/2004/14, 24 pages.

Bisilliat, J., 1996, Femmes du sud, chefs de famille, Paris, karthala, 410 pages.

Bisilliat J., 1983, «The Feminine Sphere in the Institutions of the SonghayZarma», pp.99-106. In Femanle and Male in West Africa, Edited by Oppong C.

Blanchet, D., 1992, «Interpréter les évolutions temporelles de l'activité féminine Et de la fécondité», Population 47, (2):389-408.

Chari, U., 2000, «Le nouveau visage de l'émancipation économique des femmes», pp. 119-130. In Femmes «bâtisseurs» d'Afrique, Sous la direction de Ferera, $\mathrm{L}$

Clignet, R., 1972, «Quelques remarques sur le rôle des femmes africaines en milieu urbain : Le cas du Cameroun» Canadian Journal of African Studies 6, (2) :303-315.

Dial, F.B., 2001, «Le divorce, source de promotion de la femme ? L'exemple des femmes divorcées de Dakar et de Saint-Louis ( Sénégal).» Paper presented for : Gen re, Population e $t$ Développement en Afrique. Colloque International, Abidjan, 
Mahamane Ibrahima: Facteurs d'Évolution de la Contribution Économique des Femmes au Niger

j uillet, 15 pages.

Dunbar R.A. and Djibo H., 1992, Islamic, Public Policy and the Legal Status of Women in Niger. Genesys Special Study \#12, USAID, Office of Women in Development, March, 38 p.

Dupire, M., 1987, «Situation de la femme dans une société pastorale (Peul Wodaabe, nomades du Niger)», pp. 51-91. In Femme d'Afrique Noire, Edited by Paulme, D.

Fapohunda E., 1983, «Female and Male Work Profiles», pp. 32-53. In Female and Male in West Africa, Edited by Oppong, $\mathrm{C}$.

FeDDAF, 2004, Rapport de synthèse des ONG d'Afrique de l'Ouest sur la mise en cuvre de la plateforme d'action de Beijing. Bureau Sous-régional, Afrique de l'Ouest, Lomé, Togo, 27 Août, 36 pages.

Kempeneers M., 1992, Le travail au féminin: Analyse démographique de la discontinuitéprofessionnelle des femmes au Canada. Les presses de l'Université de Montréal, 216 pages.

Nunez, A., 1989, «Appui à des groupements féminins», pp. 233253. In Des coopératives témoignent de leur expérience avec des femmes au Sahel, Sous la direction de Champagne.
Rexroat C., 1990, «Race and Marital Status Differences in the Labor Force Behavior of Female Family Heads : The Effects of Household Structure.» In Journal of Marriage and the Family 52, (3): 591-601.

Schildkrout E., 1983, «Dependence and Autonomy: The Economic Activities of Secluded Hausa Women in Kano», pp. 107-126. In Female and Male in West Africa, Edited by Oppong, $\mathrm{C}$.

Schwarz A., 1972, "Illusion d'une émancipation et aliénation réelle de 'ouvrière zairoise» Canadian Journal of African Studies 6, (2) : 183-212, Special Issue.

Sow, F., 2000, «Reculer les frontières de l'inégalité : obstacles et contraintes à la participation des femmes au développement», pp. 193-205. In Femmes «bâtisseurs» d'Afrique, Sous la direction de Ferara, $\mathrm{L}$.

Unifem, 2005, Progress of the World's Women 2005: Women, Work and Poverty,110 p.

United Nations, 2001, Convention on the Elimination of All Forms of Discrimination against Women: Niger, 69 p.

Véron, J., 1988, «Activité féminine et structures familiales. Quelle dépendance ?» Population 43, (1) : 103-120. 
African Population Studies Vol. $22 n^{\circ}$ 2/Etude de la Population Africaine Vol. $23 N^{*} 1$

World Bank, 1999, Inégalité des sexes, croissance et réduction de la pauvreté, FIndings No. 129, décembre, Région Afrique. 